\title{
3d-orbital occupancy regulated Ir-Co atomic pair toward superior bifunctional oxygen electrocatalysis
}

Meiling Xiao ${ }^{1} \ddagger$, Jianbing Zhu ${ }^{1} \ddagger$, Shuang $\mathrm{Li}^{1}+$, Gaoran $\mathrm{Li}^{1}$, Wenwen Liu ${ }^{1}$, Ya-Ping Deng ${ }^{1}$, Zhengyu Bai ${ }^{*}$, Lu $\mathrm{Ma}^{3}$, Ming Feng ${ }^{4}$, Tianpin $\mathrm{Wu}^{3}$, Dong $\mathrm{Su}^{5}$, Jun $\mathrm{Lu}^{6}$, Aiping $\mathrm{Yu}^{1}$ and Zhongwei $\mathrm{Chen}^{1 *}$

1 Department of Chemical Engineering, Waterloo Institute for Nanotechnology, Waterloo Institute for Sustainable Energy, University of Waterloo, Waterloo, Ontario N2L 3G1, Canada

2 Collaborative Innovation Center of Henan Province for Green Manufacturing of Fine Chemicals, Key Laboratory of Green Chemical Media and Reactions, Ministry of Education, School of Chemistry and Chemical Engineering and College of Physics and Materials Science, Henan Normal University, Xinxiang, Henan, 453007 P. R. China

3 X-Ray Science Division, Argonne National Laboratory, Lemont, IL, 60439, USA

4 Key Laboratory of Functional Materials Physics and Chemistry of the Ministry of Education, Jilin Normal University, Changchun, 130103 China

5 Beijing National Laboratory for Condensed Matter Physics, Institute of Physics, Chinese Academy of Sciences, Beijing, 100190, China

6 Chemical Sciences and Engineering Division, Argonne National Laboratory, Lemont, IL, 60439, USA

Corresponding author email address: zhwchen@uwaterloo.ca (Z. Chen); baizhengyu@htu.cn (B. Yu) 
Synthesis of Precursors. For the synthesis of pristine Zn-ZIF, $6.5 \mathrm{~g}$ 2-methylimidazole was dissolved in 80 $\mathrm{mL}$ methanol with stirring. Then $40 \mathrm{~mL}$ methanol containing $3 \mathrm{~g} \mathrm{Zn}\left(\mathrm{NO}_{3}\right)_{2} \cdot 6 \mathrm{H}_{2} \mathrm{O}$ was added with vigorous stirring for $24 \mathrm{~h}$ at room temperature. The obtained product was separated by centrifugation and washed with methanol and finally dried at $60^{\circ} \mathrm{C}$ under vacuum for overnight. While for the ZnCo-ZIF, Ir-Zn-ZIF and Ir$\mathrm{ZnCo}-\mathrm{ZIF}$, the procedure was similar as that of $\mathrm{Zn}$-ZIF, expect that a certain amount of metal salts $\left(\mathrm{Zn}\left(\mathrm{NO}_{3}\right)_{2} \cdot 6 \mathrm{H}_{2} \mathrm{O}, \operatorname{Ir}(\mathrm{acac})_{3}\right)$ were added with $\mathrm{Zn}\left(\mathrm{NO}_{3}\right)_{2} \cdot 6 \mathrm{H}_{2} \mathrm{O}$ in the feeding solution.

Synthesis of the Pyrolyzed Catalysts. The power of the as-obtained precursors was transferred into a ceramic boat and placed in a tube furnace. The sample was pyrolyzed under flowing $\mathrm{Ar} / \mathrm{H}_{2}\left(10 \% \mathrm{H}_{2}\right)$ gas at $1050^{\circ} \mathrm{C}$ for $1 \mathrm{~h}$ and then naturally cooled to room temperature. They are denoted as N-C, Co-N-C, Ir-N-C and IrCo-N-C, which were derived from Zn-ZIF, ZnCo-ZIF, Ir-Zn-ZIF and Ir-ZnCo-ZIF, respectively.

Physical Characterization. Scanning electron microscopy (SEM) measurements were performed with an XL 30 ESEM FEG field emission scanning electron microscope. Transmission electron microscopy (TEM), high resolution transmission electron microscopy (HRTEM), high-annular dark-field scanning transmission electron microscopy (STEM) and element mapping analysis were conducted on JEOL JEM-2100F. The high-angle annular dark-field scanning transmission electron microscopy (HAADF-STEM) images were imaged by using a Hitachi HD2700C equipped with a probe spherical aberration corrector at Brookhaven National Laboratory. X-ray diffraction (XRD) measurements were performed using a Rigaku MiniFlex 600 $\mathrm{X}$-ray diffractometer equipped with a $\mathrm{Cu} \mathrm{K \alpha}$ irradiation. The textural and morphological features of the catalysts were determined by nitrogen physisorption at $77 \mathrm{~K}$ in a Micromeritics ASAP 2020. Textural properties such as the specific surface area pore volume and pore size distribution were calculated from each corresponding nitrogen adsorption-desorption isotherm, applying the Brunauer-Emmett-Teller (BET) equation and the Barrett-Joyner-Halenda (BJH). X-ray photoelectron spectroscopy (XPS) measurements were carried out on $\mathrm{Mg} \mathrm{K} \alpha$ radiation source (Kratos XSAM-800 spectrometer). The bulk compositions were evaluated by inductively coupled plasma optical emission spectrometer (X Series 2, Thermo Scientific USA). Ir L-edge, Co K-edge X-ray absorption spectra were performed on the 9BM-C beam line at Advanced Photon Sources at Argonne National Laboratory. The EXAFS raw data were then background-subtracted, normalized and Fourier transformed by the standard procedures with the IFEFFIT package ${ }^{1}$.

Electrochemical Measurement. All electrochemical measurements were conducted in a conventional three-electrode cell at room temperature $\left(\sim 25^{\circ} \mathrm{C}\right)$ using the 750B Bipotentiostat (CH Instruments). The asprepared Ir-based catalyst ink was prepared by ultrasonically dispersing $5 \mathrm{mg}$ catalyst in a suspension containing $50 \mu \mathrm{L}$ Nafion ( $5 \mathrm{wt} \%$ ) solution and $950 \mu \mathrm{L}$ ethanol. As for commercial Pt/C (46.6 wt\% Pt, TKK), $2 \mathrm{mg}$ catalyst was dispersed ultrasonically in a mixture solution containing $30 \mu \mathrm{l}$ Nafion (5 wt \%) solution, $500 \mu \mathrm{L}$ isopropanol and $1500 \mu \mathrm{L}$ Milli-Q water. The catalyst film coated electrode was obtained by dispersing the catalyst ink on a glassy carbon rotating ring-disk electrode followed by drying in air. The catalyst loadings on RRDE were $0.4 \mathrm{mg} \mathrm{cm}^{-2}$ for as-prepared catalysts, and $40 \mu \mathrm{g}_{\mathrm{Pt}} \mathrm{cm}^{-2}$ for Pt/C-TKK catalyst.

The electrolyte was $0.1 \mathrm{M} \mathrm{KOH}$ solution for both ORR and OER measurements. RDE measurements for ORR were conducted by liner sweep voltammetry (LSV) from $1.0 \mathrm{~V}$ to $0 \mathrm{~V}$ at a scan rate of $5 \mathrm{mV} \mathrm{s}^{-1}$ at 900 $\mathrm{rpm}$, while the potential window for OER was $1.1 \mathrm{~V}$ to $1.8 \mathrm{~V}$. All the current density presented in the figures is normalized to the electrode surface area with $85 \%$ iR-correction. 
The ORR stability was investigated by continuous potential cycling in oxygen-saturated $0.1 \mathrm{M} \mathrm{KOH}$ solution between $0.6 \mathrm{~V}$ and $1.0 \mathrm{~V}$ with the scan rate at $0.1 \mathrm{~V} \mathrm{~s}^{-1}$. And after 10000 cycles, the ORR steady-state polarization measurements were conducted in $\mathrm{O}_{2}$-saturated $0.1 \mathrm{M} \mathrm{KOH}$ solution with scanning rates of 5 $\mathrm{mV} \mathrm{s}^{-1}$ and rotation rate at $900 \mathrm{rpm}$.

Zn-air Battery Measurements. For the Zn-air battery test, the air electrode was prepared by uniformly coating the as-prepared catalyst ink onto carbon paper then drying it at $80^{\circ} \mathrm{C}$ for $4 \mathrm{~h}$. The catalyst loading was $1 \mathrm{mg} \mathrm{cm}^{-2}$. A $\mathrm{Zn}$ plate was used as the anode. Both electrodes were assembled into a $\mathrm{Zn}$-air battery, and $6 \mathrm{M} \mathrm{KOH}$ aqueous solution with $0.2 \mathrm{M} \mathrm{Zn}\left(\mathrm{CH}_{3} \mathrm{COO}\right)_{2}$ was used as the electrolyte. The galvanodynamic charge and discharge profiles of the battery were obtained by scaling the current density from 0 to $250 \mathrm{~mA}$ $\mathrm{cm}^{-2}$. The cycling tests were conducted at a current density of $5 \mathrm{~mA} \mathrm{~cm}-2$ with 15 min discharge and $15 \mathrm{~min}$ charge time for each cycle. The tests were operated in ambient air condition.

DFT calculations. The calculations were carried out using density functional theory (DFT) with the PBE+U method (effective $\mathrm{U}$ of 4 and $2 \mathrm{eV}$ for Co and Ir, respectively) ${ }^{2}$. The Vienna ab-initio simulation package (VASP) ${ }^{3-6}$ was employed. The plane wave energy cutoff was set as $400 \mathrm{eV}$. The Fermi scheme was employed for electron occupancy with an energy smearing of $0.1 \mathrm{eV}$. The first Brillouin zone was sampled in the Monkhorst-Pack grid ${ }^{7}$. The $4 \times 4 \times 1$ k-point mesh is employed in our calculation. The energy (converged to $1.0 \times 10^{-6} \mathrm{eV} /$ atom) and force (converged to $0.03 \mathrm{eV} / \AA$ ) were set as the convergence criterion for geometry optimization. The spin polarization was considered in all calculation. The D3 correction method is employed to describe the van der Waals interactions ${ }^{8}$. 

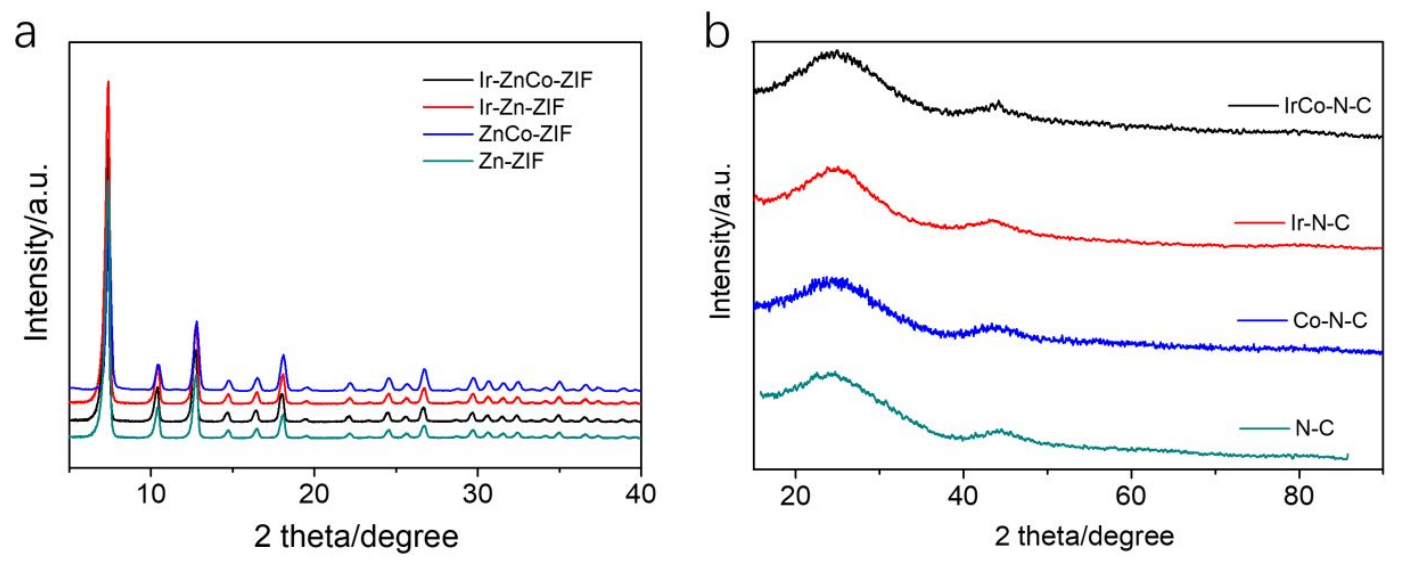

Figure S1. XRD patterns of (a) ZIF precursors and (b) pyrolyzed catalysts.
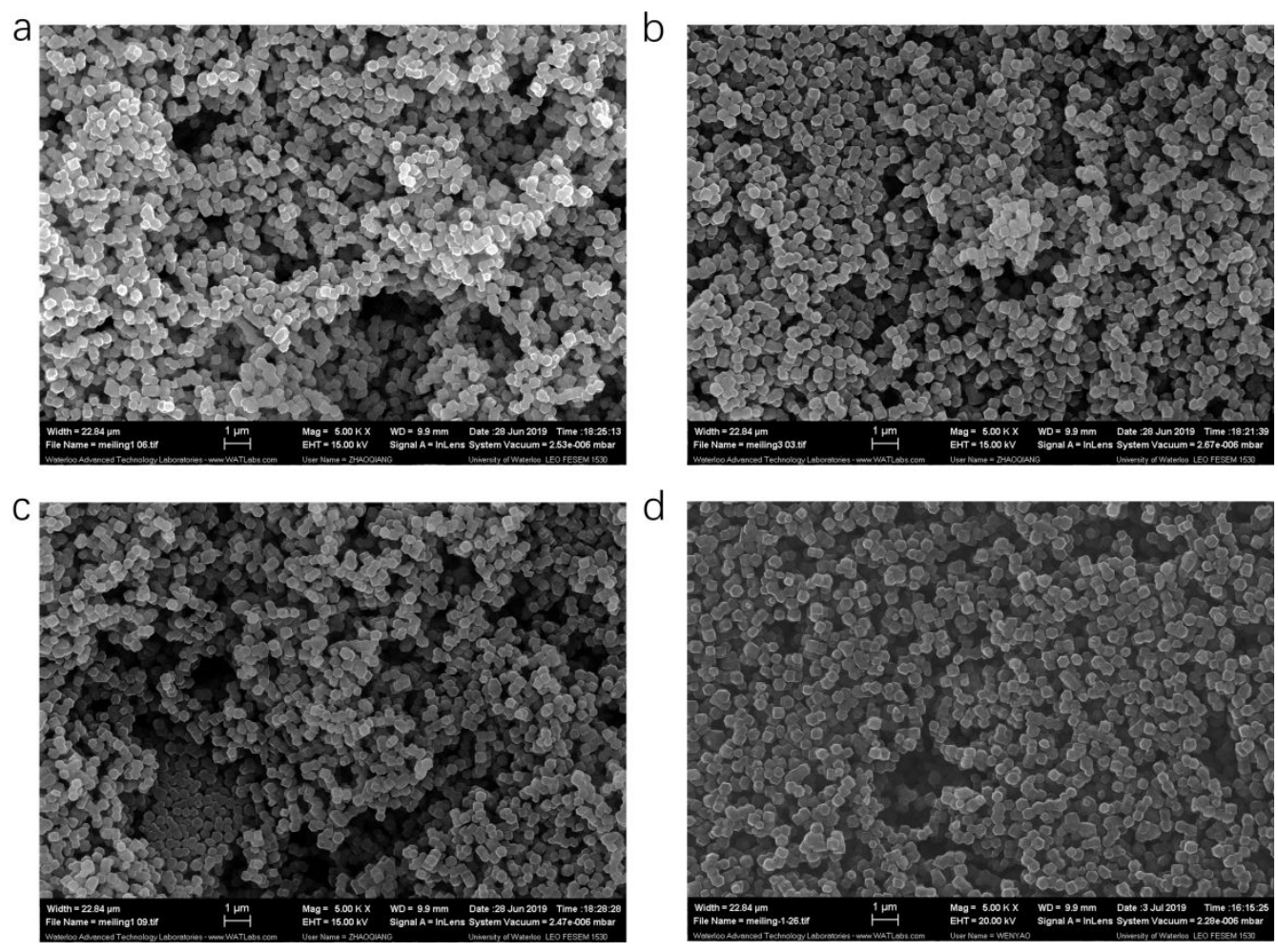

Figure S2. STM images for (a) Zn-ZIF, (b) ZnCo-ZIF, (c) Ir-Zn-ZIF, (d) Ir-ZnCo-ZIF. 

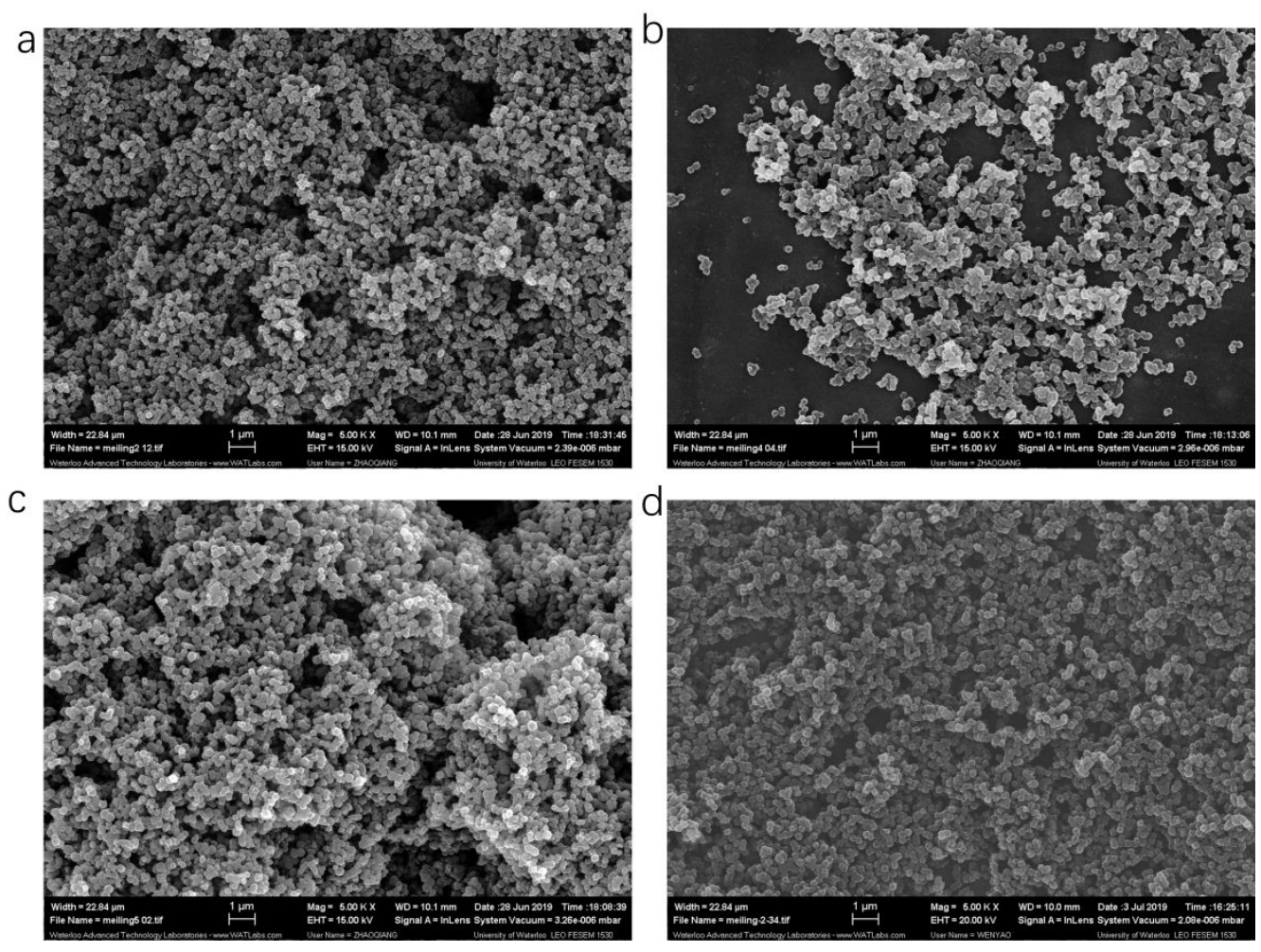

Figure S3. STM images for (a) N-C, (b) Co-N-C, (c) Ir-N-C, (d)IrCo-N-C.
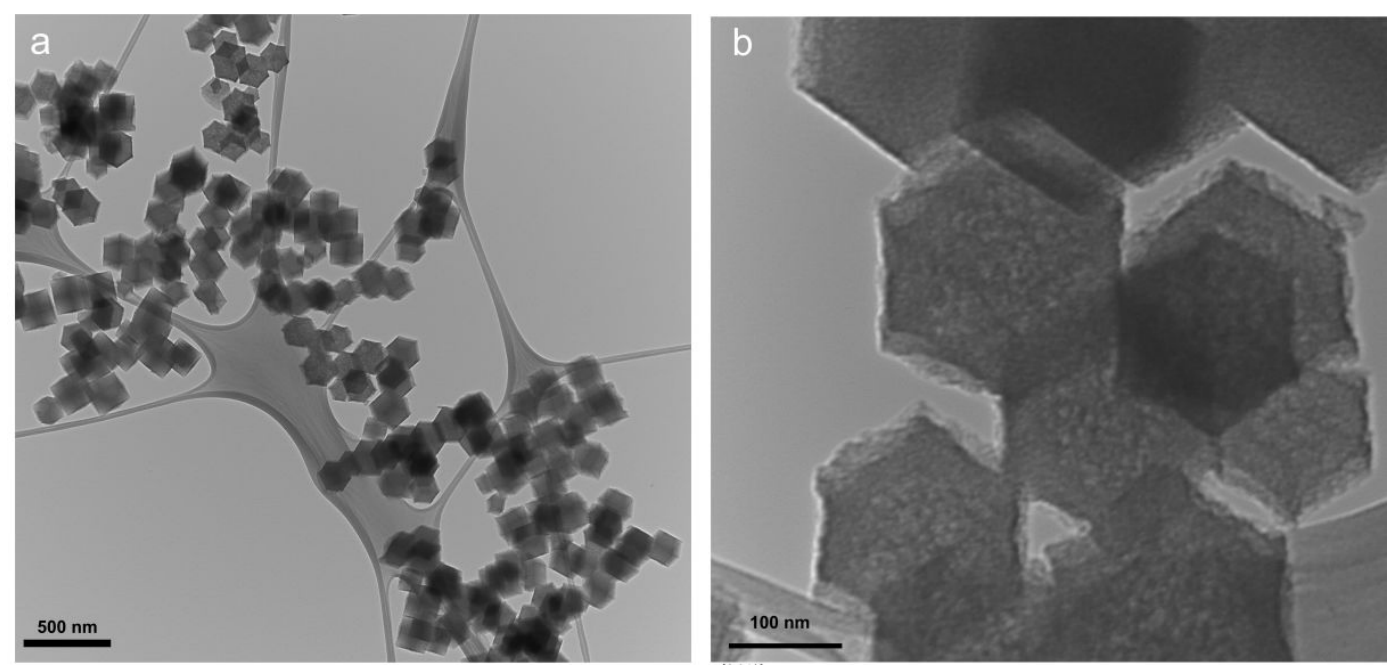

Figure S4. TEM images for IrCo-N-C. 

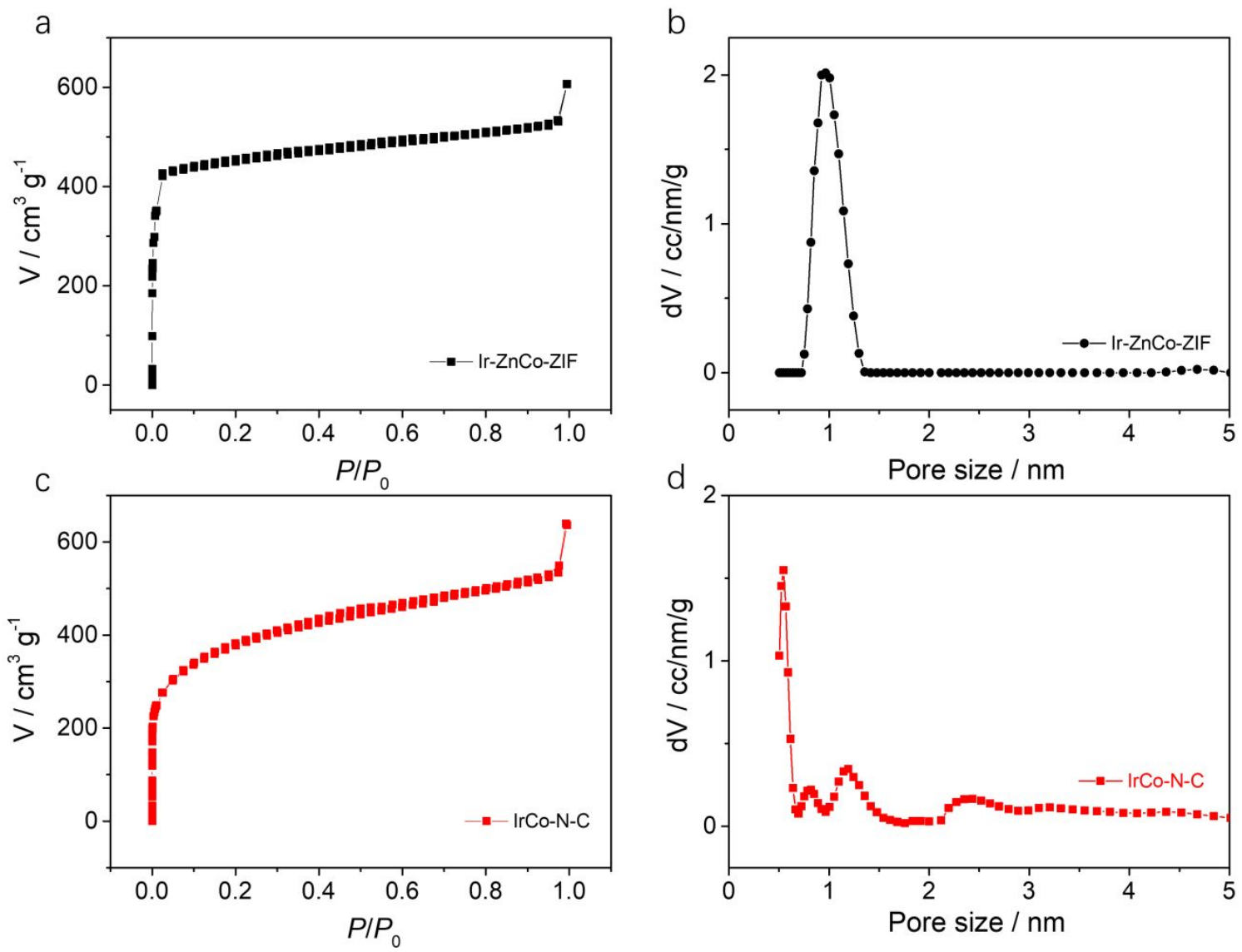

Figure S5. $\mathrm{N}_{2}$ adsorption-desorption isotherms for ZIFs and the corresponding pore size distribution curves. 

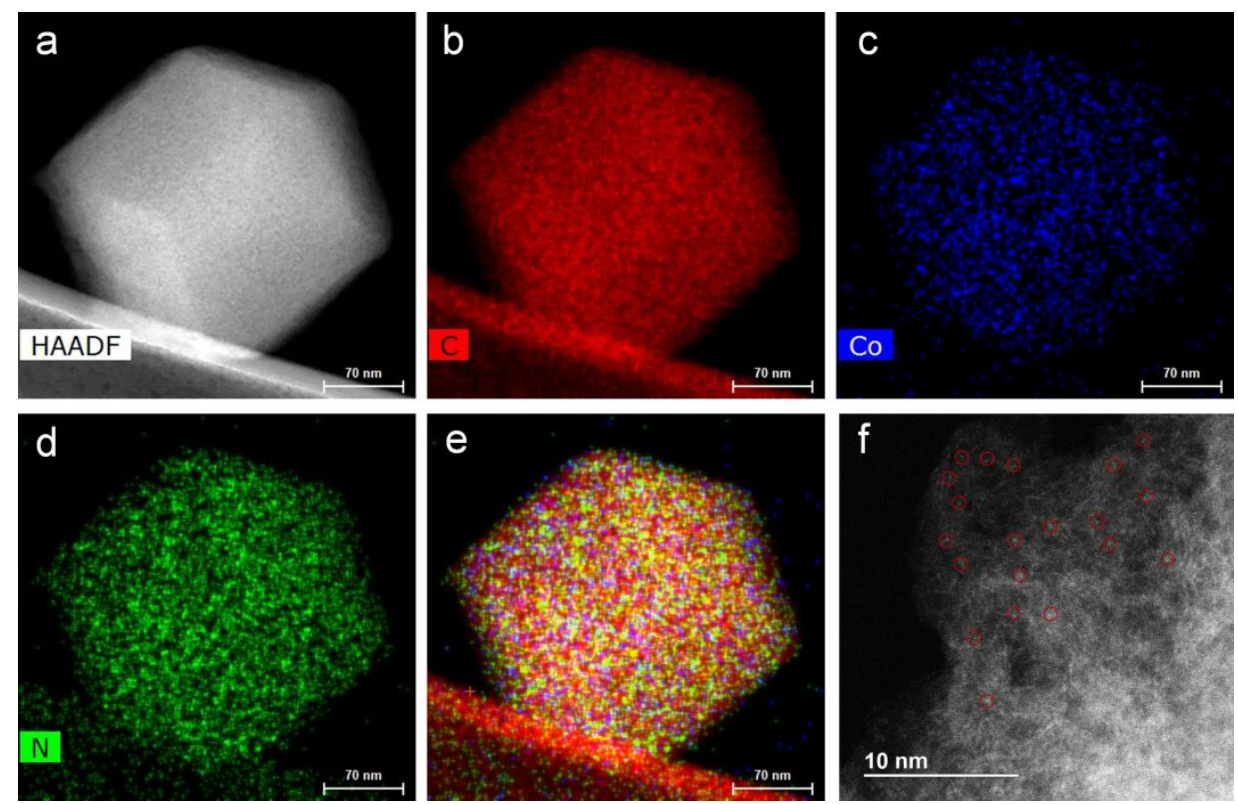

Figure S6. (a) STEM, (b-e) elemental mapping and (f) Aberration-corrected high-resolution HAADF-STEM image of Co-N-C, with the distinct bright dots indicating Co is atomically dispersed on the nitrogen doped carbon matrix.
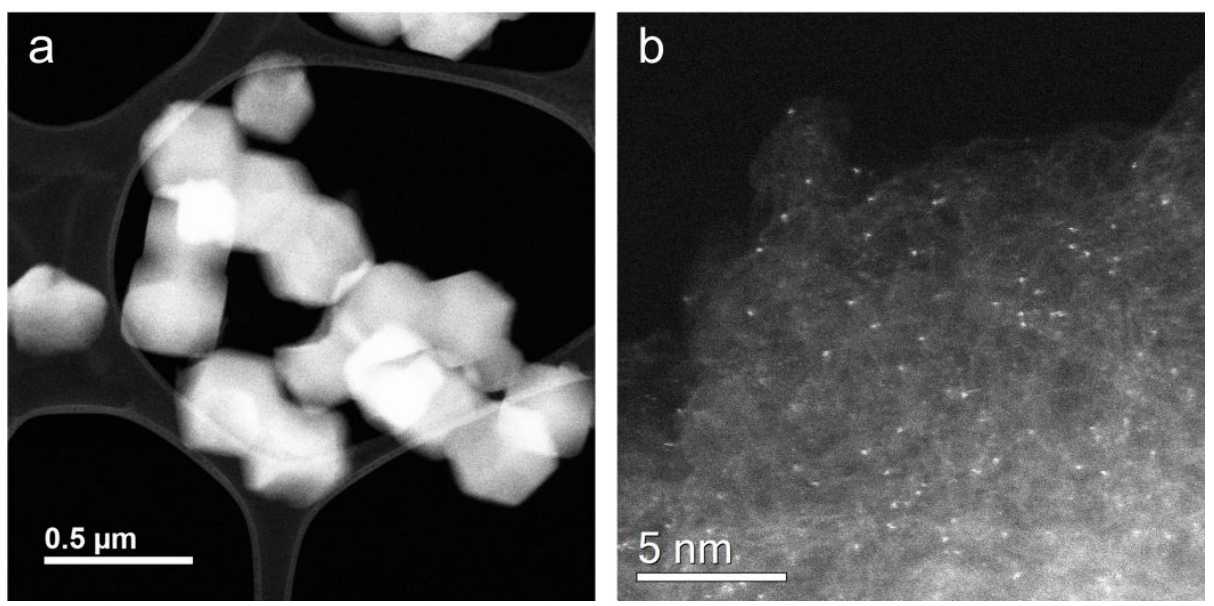

Figure S7. (a) STEM and (b) Aberration-corrected high-resolution HAADF-STEM image of Ir-N-C, with the distinct bright dots indicating Ir is atomically dispersed on the nitrogen doped carbon matrix. 

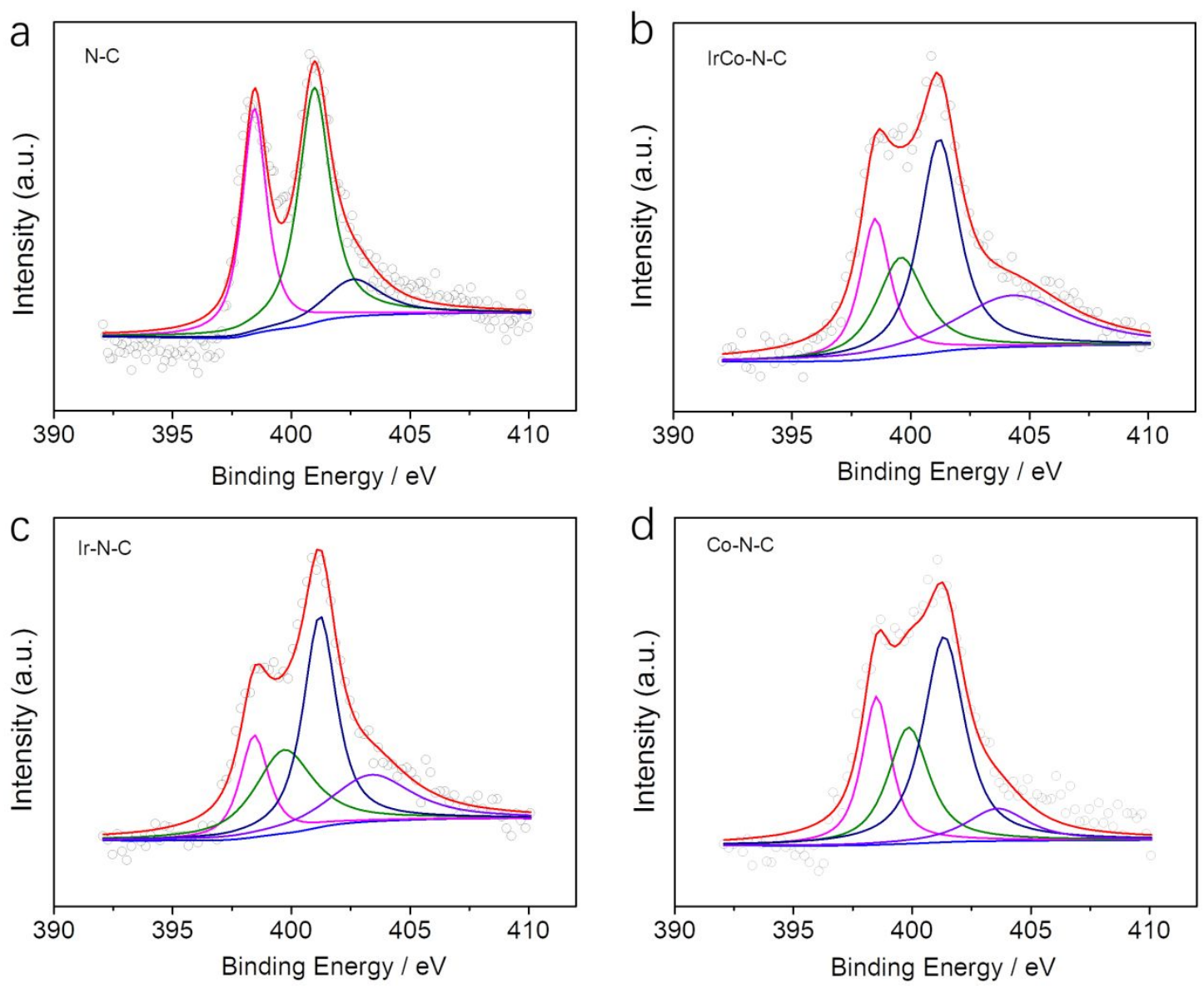

Figure S8. High-resolution N 1s XPS spectra for (a) N-C, (b) IrCo-N-C, (c) Ir-N-C and (d) Co-N-C.

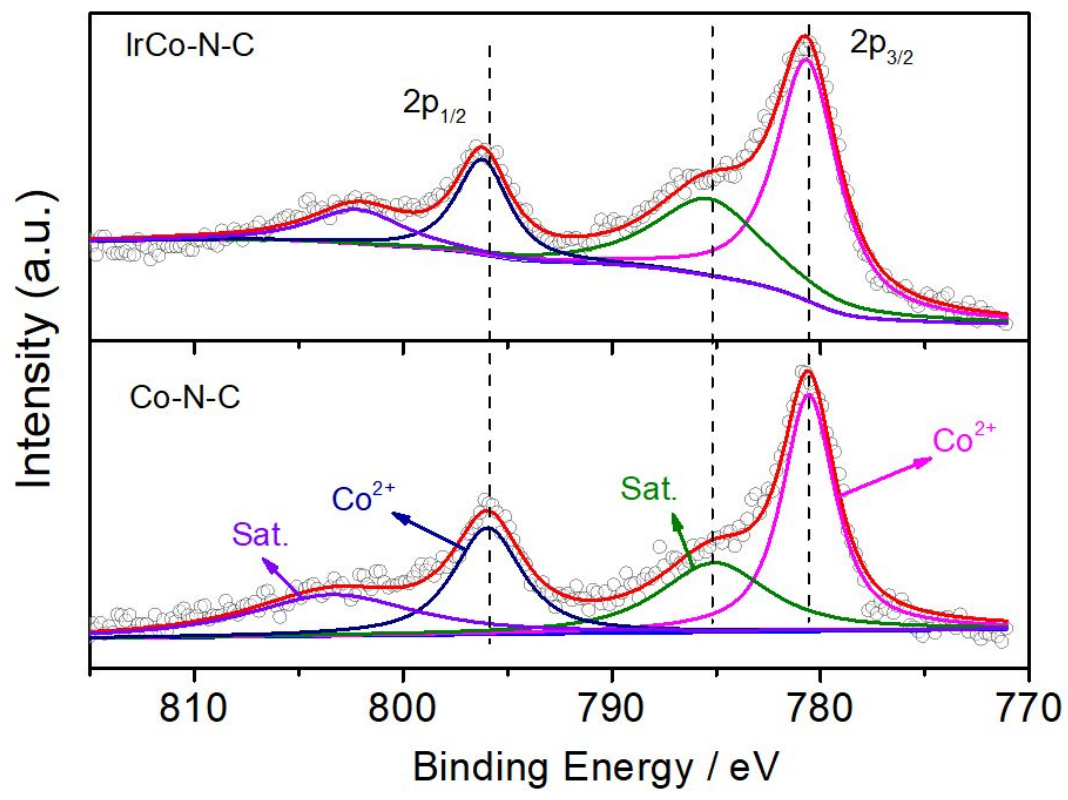

Figure S9 High-resolution Co 2p XPS spectra for IrCo-N-C and Co-N-C. 


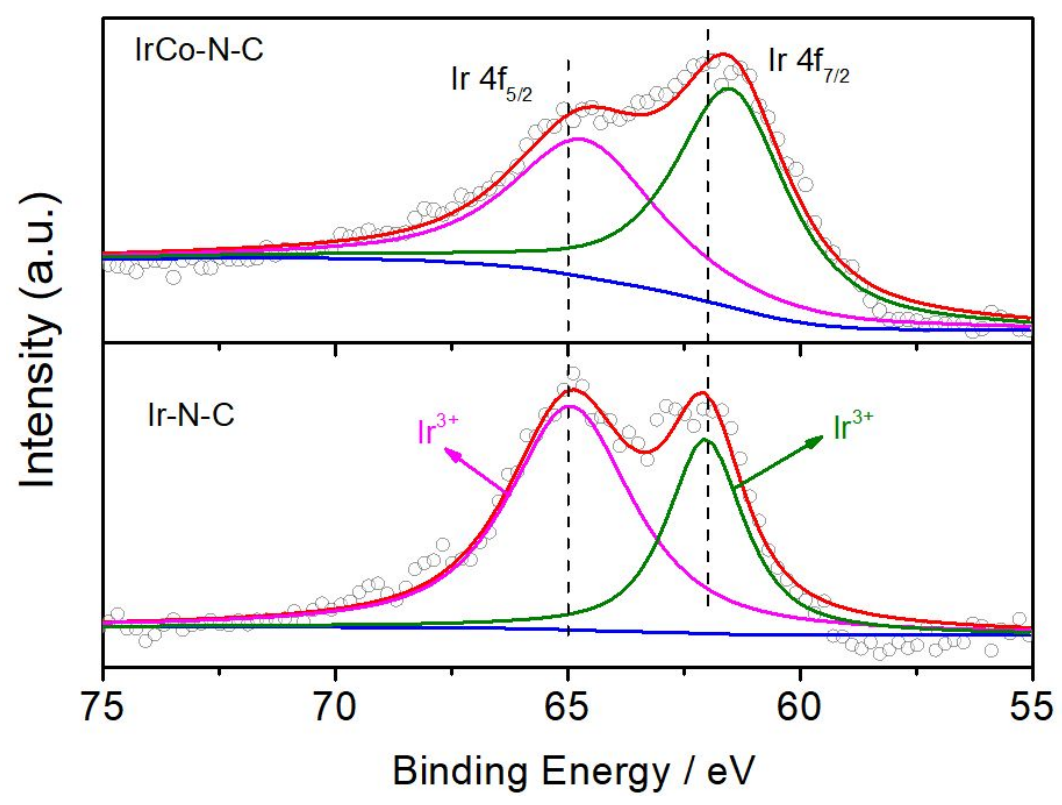

Figure S10 High-resolution Ir 4f XPS spectra for IrCo-N-C and Ir-N-C.

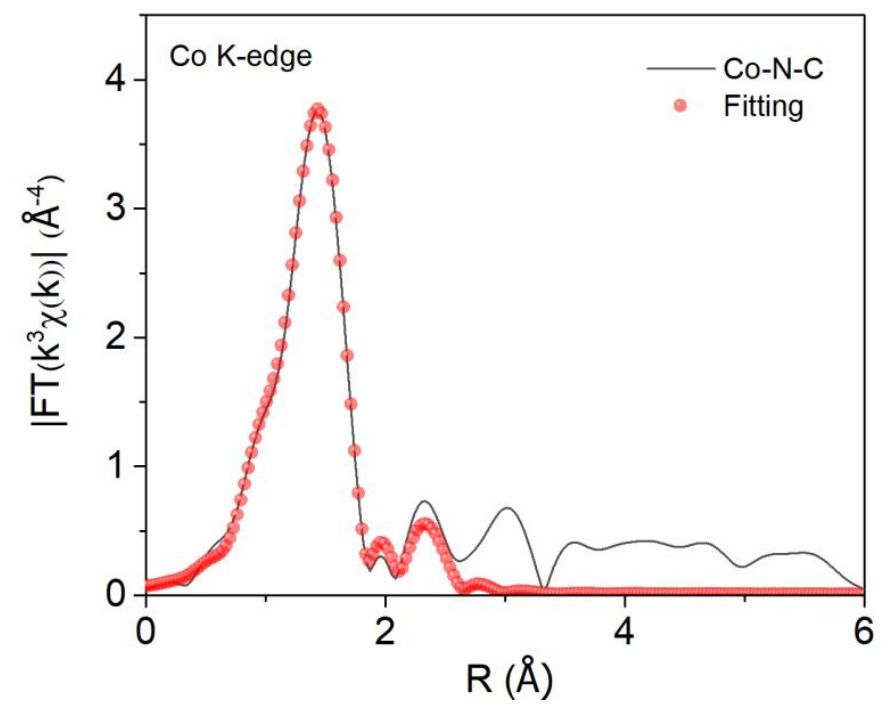

Figure S11 EXAFS fitting curves of Co-N-C at Co K-edge. 


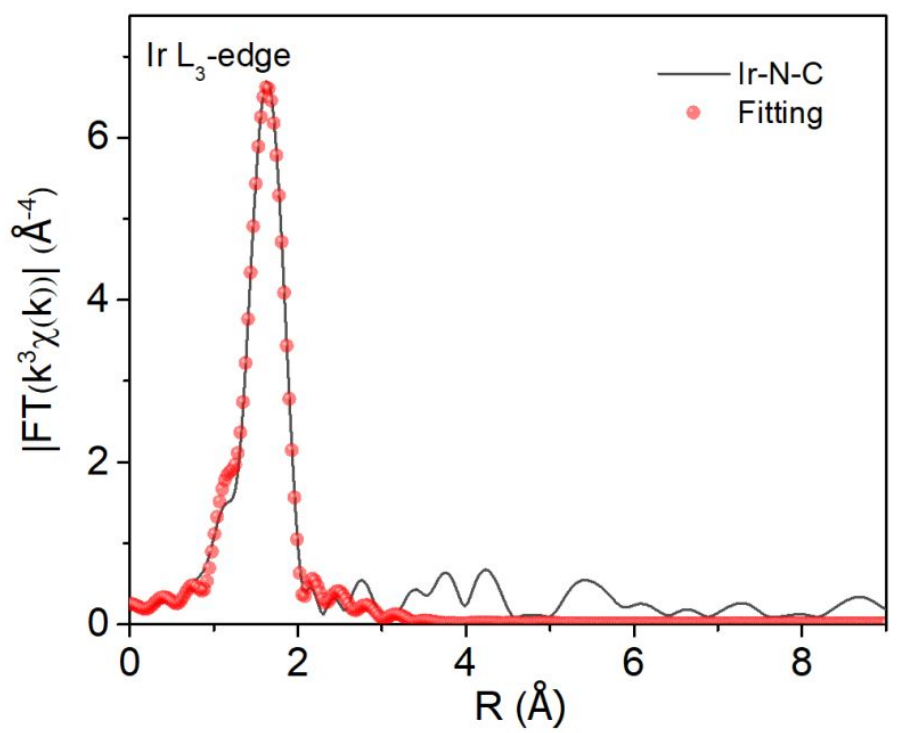

Figure S12 EXAFS fitting curves of Ir-N-C at Ir $\mathrm{L}_{3}$-edge. 


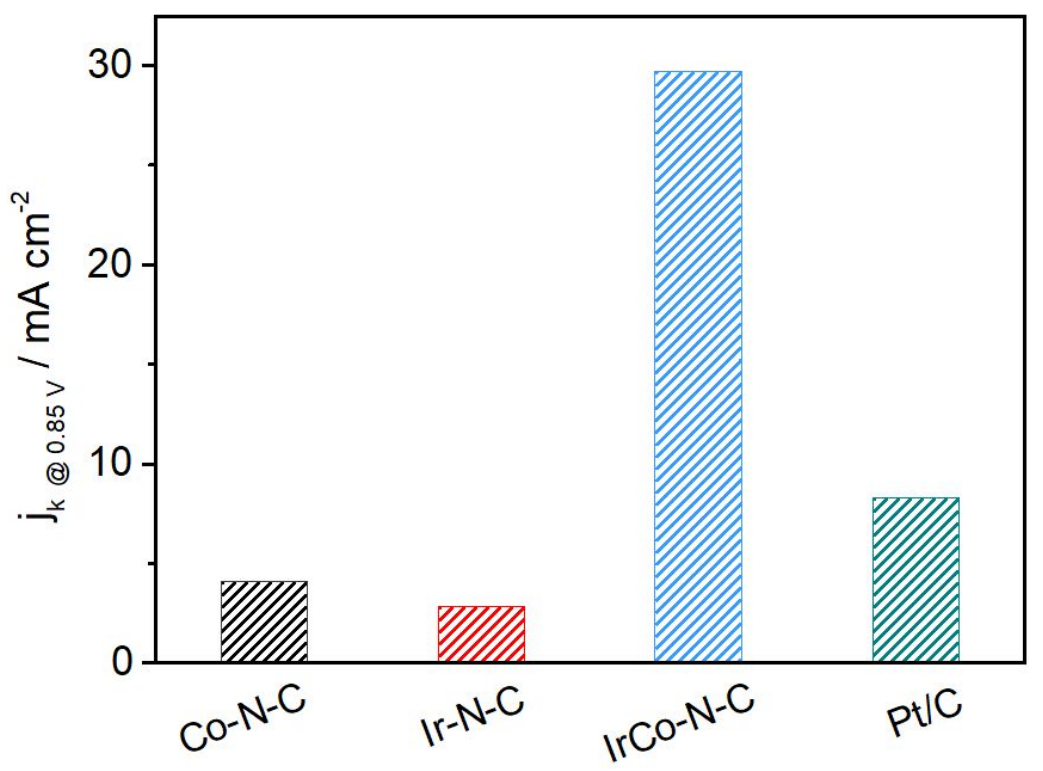

Figure S13 Kinetic current density at $0.85 \mathrm{~V}$ on various catalysts.

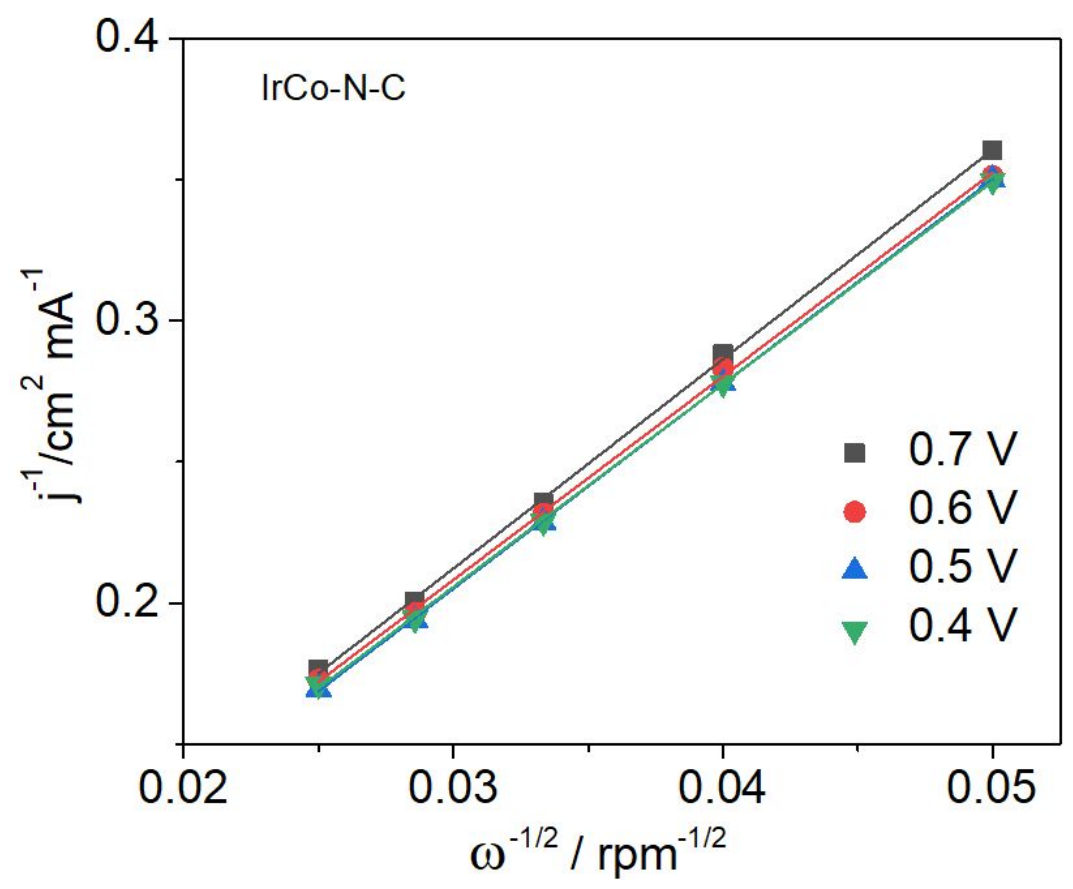

Figure S14 K-L plots of IrCo-N-C. 

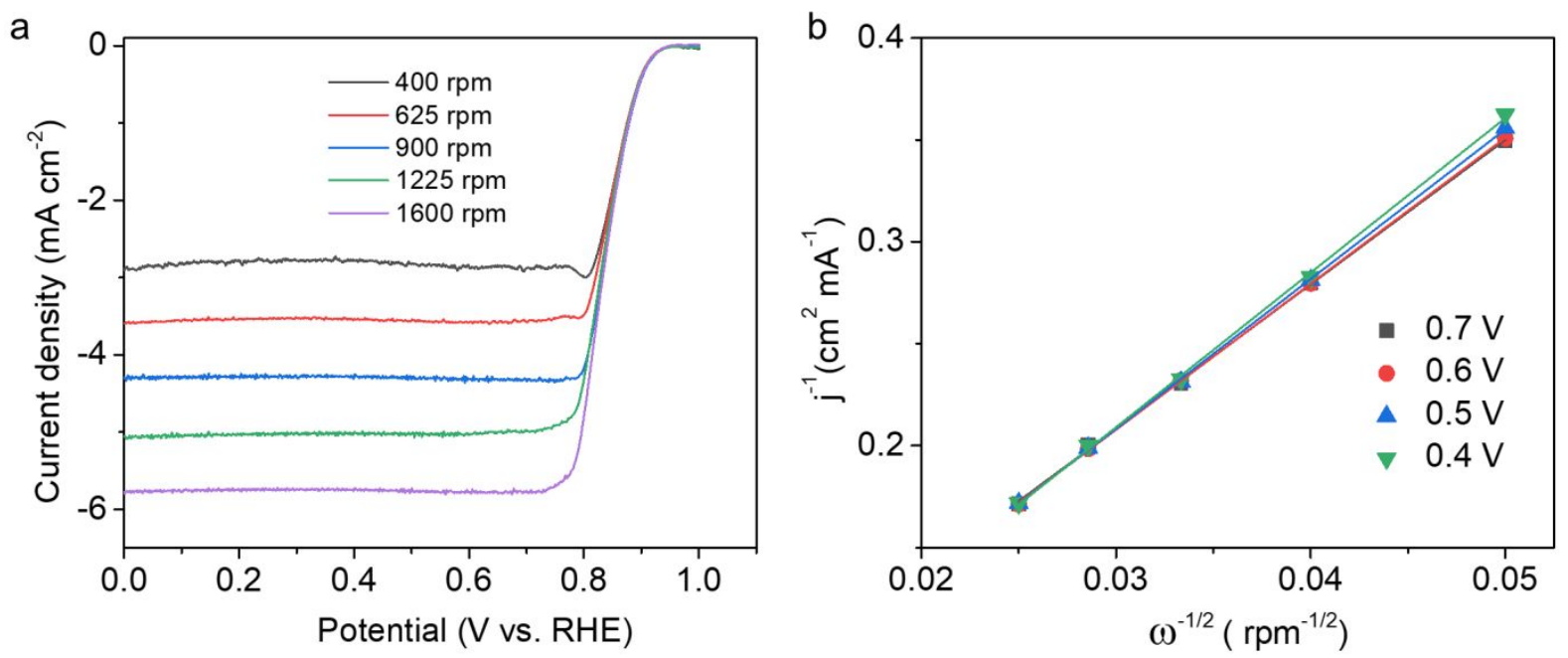

Figure S15 ORR polarization curves of Co-N-C at different rotation speeds and the corresponding K-L plots.
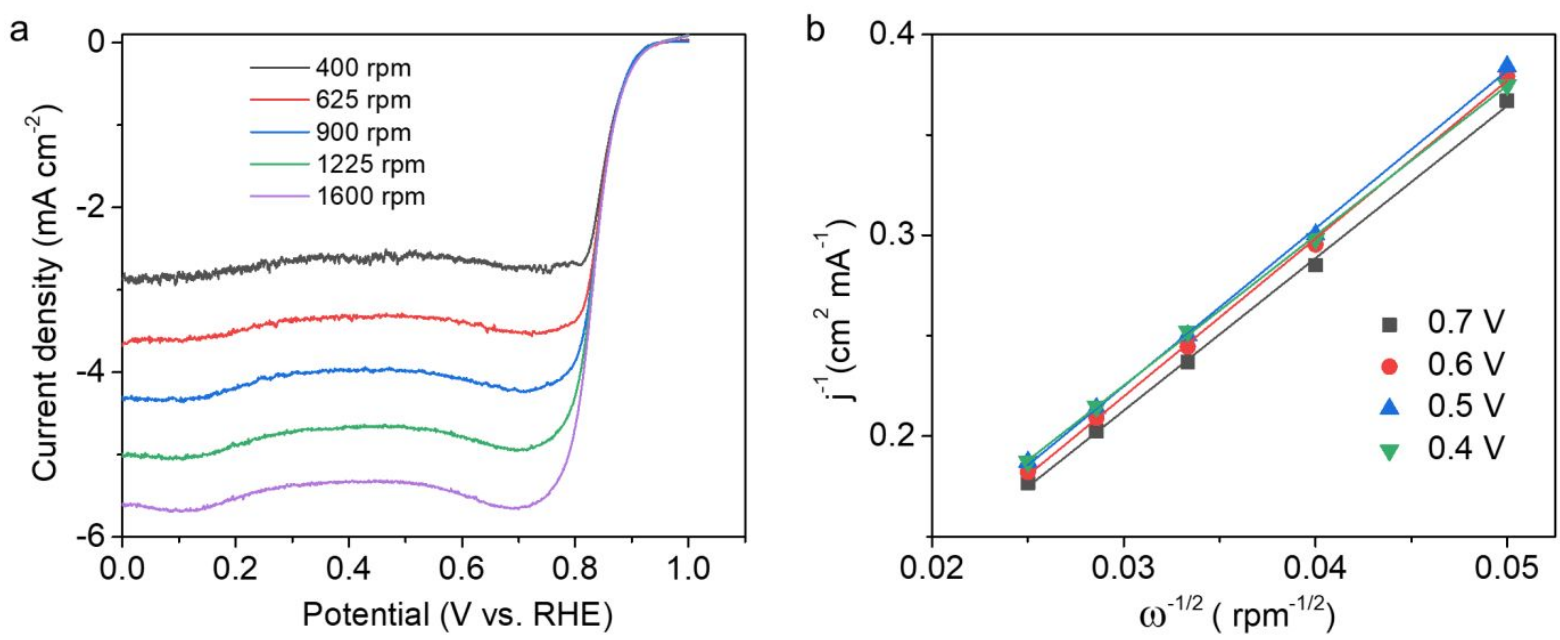

Figure S16 ORR polarization curves of Ir-N-C at different rotation speeds and the corresponding K-L plots.

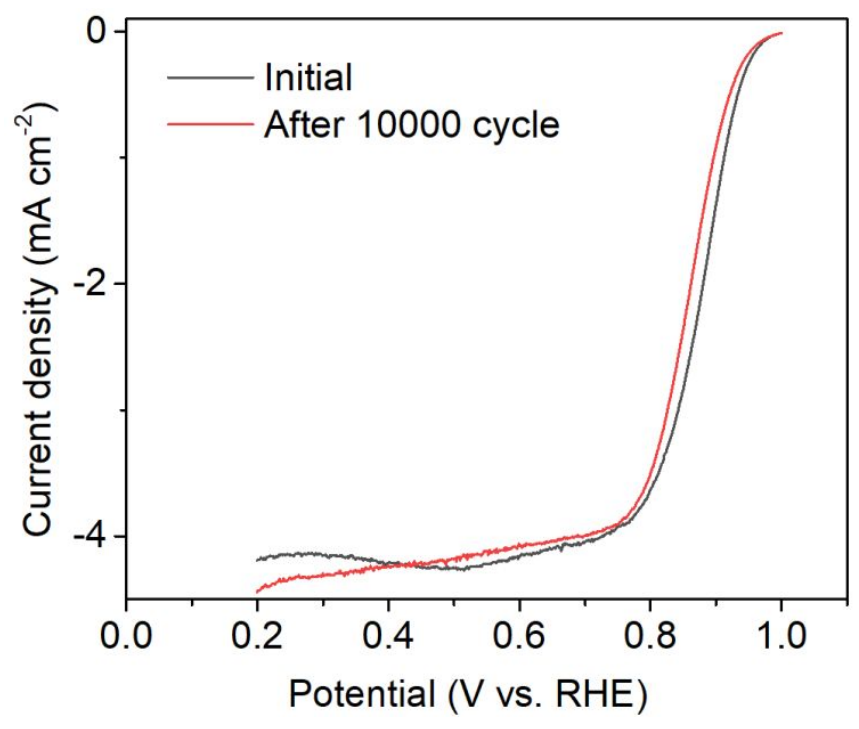

Figure S17 ORR polarization curves of $\mathrm{Pt} / \mathrm{C}$ before and after 10000 cycles operation. 
a
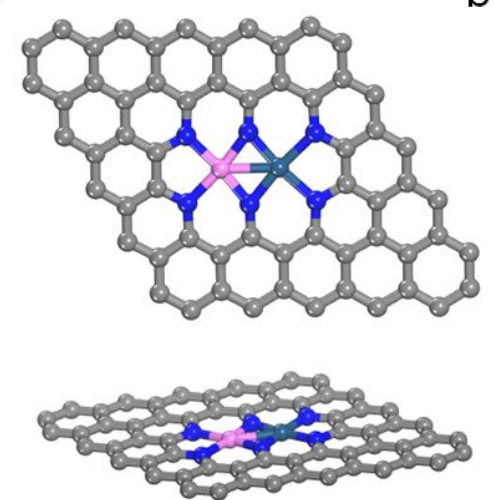

d

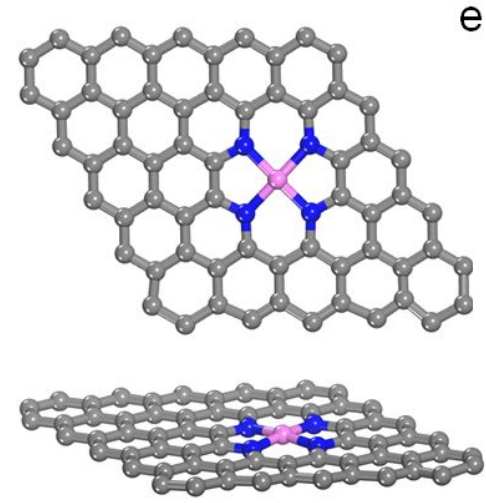

b

e
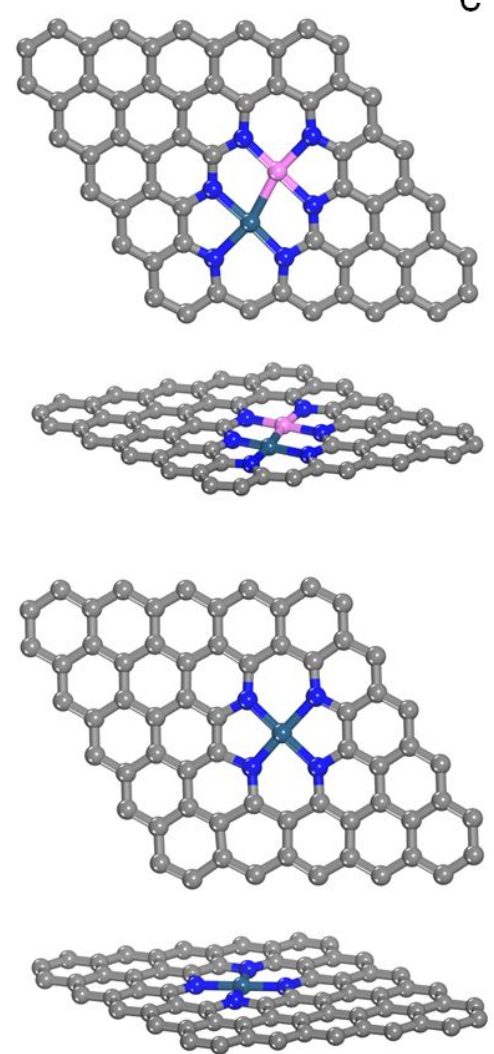
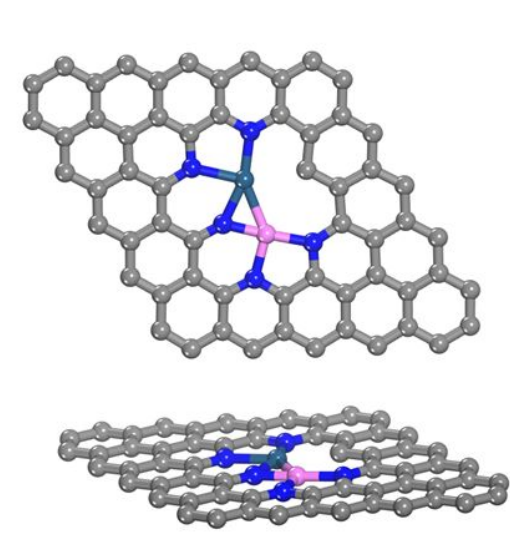

Figure S18 Optimized configurations for (a) $\operatorname{IrCoN}_{6}-1$, (b) $\operatorname{IrCoN}_{6}-2$, (c) $\operatorname{IrCoN}_{5}$, (d) $\mathrm{CoN}_{4}$ and (e) $\operatorname{IrN}_{4}$. 


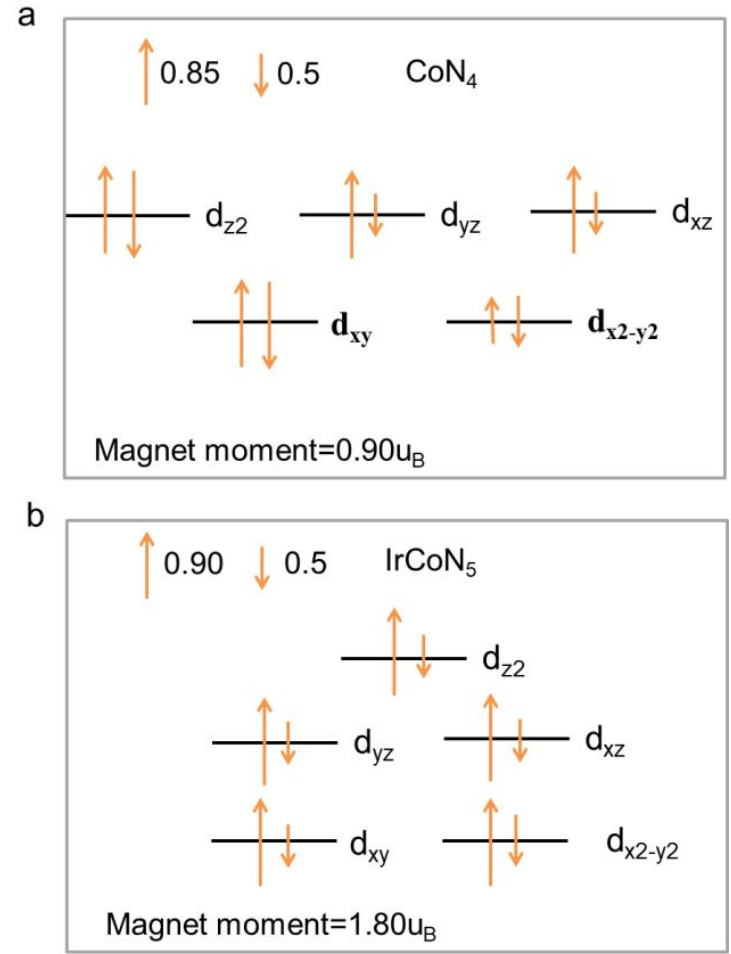

Figure S19 Co-3d electron arrangement in (a) $\mathrm{CoN}_{4}$ and (b) $\mathrm{IrCoN}_{5}$.

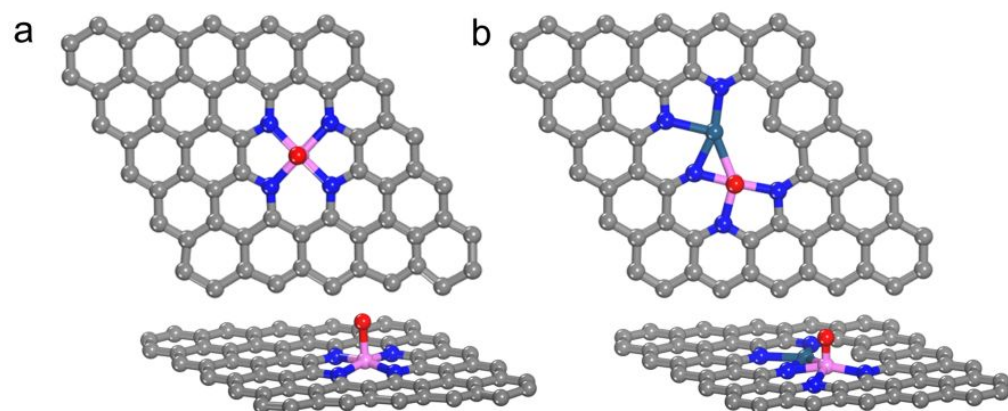

Figure S20 Optimized configurations of $\mathrm{O}$ adsorbed on (a) $\mathrm{CoN}_{4}$ and (b) $\mathrm{IrCoN}_{5}$ site. 


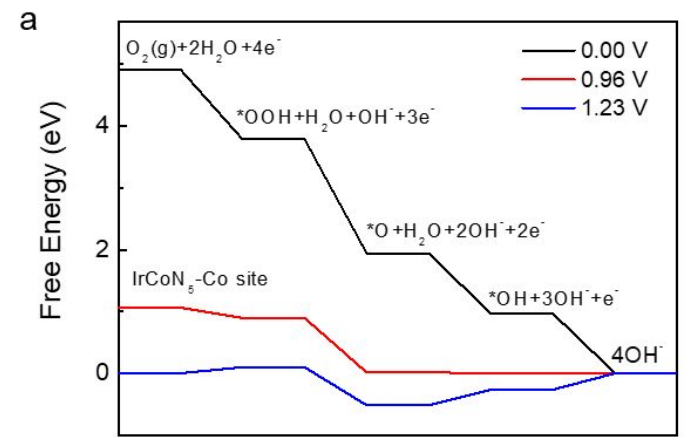

Reaction Coordinate

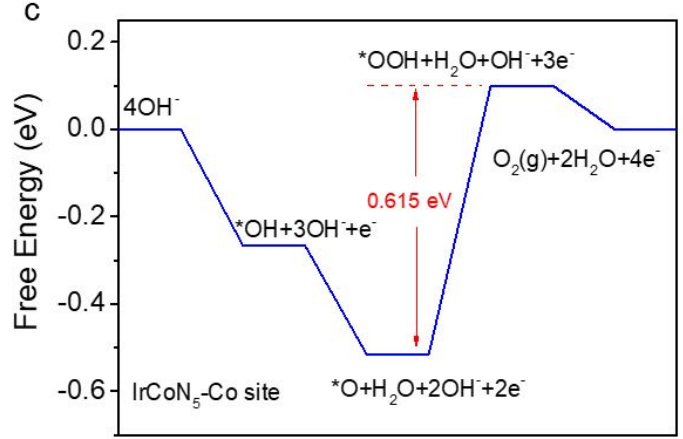

Reaction Coordinate

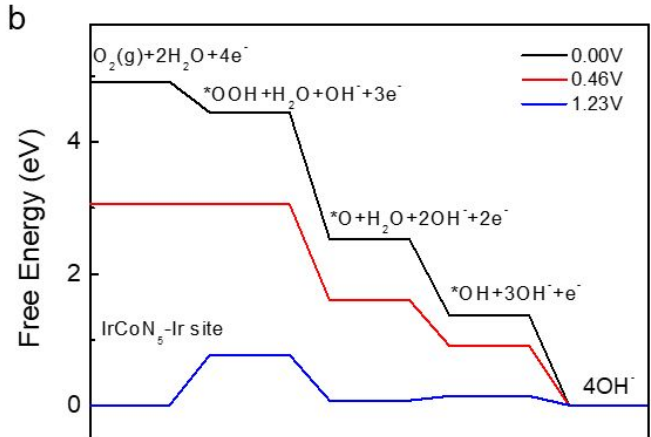

Reaction Coordinate

d

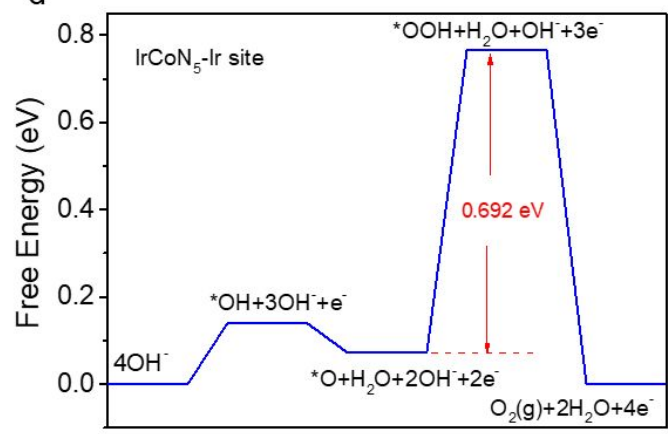

Reaction Coordinate

Figure S21 ORR Gibbs free energy profiles on (a) $\operatorname{IrCoN}_{5}$-Co site and (b) $\operatorname{IrCoN}_{5}-\mathrm{Ir}$ site at different potentials; OER Gibbs free energy profiles on (c) $\operatorname{IrCoN}_{5}-\mathrm{Co}$ site and (d) $\operatorname{IrCoN}_{5}$-Ir site at $\mathrm{U}=1.23 \mathrm{~V}$. 
Table S1. The metal element content of the synthesized catalysts.

\begin{tabular}{lll}
\hline Sample & $\operatorname{Ir}(\mathrm{wt} \%)$ & Co $(\mathrm{wt} \%)$ \\
\hline IrCo-N-C & 0.18 & 1.02 \\
Ir-N-C & 0.20 & - \\
Co-N-C & - & 1.16 \\
\hline
\end{tabular}

Table S2 Co K-edge EXAFS fitting results of the prepared catalysts

\begin{tabular}{ccccccc}
\hline Sample & Shell & $\mathrm{CN}$ & $\mathrm{R}(\AA)$ & $\sigma^{2}\left(10^{-3} \AA^{2}\right)$ & $\Delta \mathrm{E}_{0}$ & $\mathrm{R}$ factor \\
\hline Co foil & Co-Co & 12 & 2.53 & -- & -- & -- \\
\hline \multirow{2}{*}{ Co-N-C } & Co-N & $4.6 \pm 0.3$ & $1.96 \pm 0.04$ & $3.4 \pm 0.7$ & $2.9 \pm 1.0$ & 0.01627 \\
\hline \multirow{2}{*}{ IrCo-N-C } & Co-N & $3.3 \pm 0.3$ & $1.92 \pm 0.03$ & $8.1 \pm 1.5$ & $3.6 \pm 0.8$ & \multirow{2}{*}{0.01531} \\
\cline { 2 - 6 } & Co-Ir & $0.6 \pm 0.2$ & $2.30 \pm 0.03$ & $5.1 \pm 1.8$ & $5.0 \pm 1.2$ & \\
\hline
\end{tabular}

Table S3 Ir $\mathrm{L}_{3}$-edge EXAFS fitting results of the prepared catalysts

\begin{tabular}{ccccccc}
\hline Sample & Shell & $\mathrm{CN}$ & $\mathrm{R}(\AA)$ & $\sigma^{2}\left(10^{-3} \AA^{2}\right)$ & $\Delta \mathrm{E}_{0}$ & R factor \\
\hline \multirow{2}{*}{ Ir/C } & Ir-Ir & $8.8 \pm 0.1$ & $2.70 \pm 0.02$ & $3.76 \pm 0.9$ & $7.5 \pm 1.0$ & \\
\cline { 2 - 6 } & Ir-O & $1.1 \pm 0.2$ & $1.90 \pm 0.03$ & $2.90 \pm 1.0$ & $5.1 \pm 0.8$ & 0.01195 \\
\hline \multirow{2}{*}{ Ir-N-C } & Ir-N & $4.1 \pm 0.4$ & $2.01 \pm 0.02$ & $4.2 \pm 1.6$ & $5.4 \pm 1.8$ & 0.01335 \\
\hline \multirow{2}{*}{ IrCo-N-C } & Ir-N & $3.0 \pm 0.3$ & $2.09 \pm 0.03$ & $3.1 \pm 1.3$ & $8.2 \pm 1.0$ & \\
\cline { 2 - 5 } & Ir-Co & $0.8 \pm 0.1$ & $2.32 \pm 0.05$ & $6.6 \pm 2.1$ & $7.7 \pm 1.3$ & 0.01843 \\
\hline
\end{tabular}

$\mathrm{CN}$ : coordination numbers; R: bond distance; $\sigma^{2}$ : Debye-Waller factors; $\Delta \mathrm{E}_{0}$ : the inner potential correction. $\mathrm{R}$ factor: goodness of fit. $\mathrm{S}_{0}{ }^{2}$ was set to 0.742 , according to the experimental EXAFS fit of $\mathrm{Ru}$ foil reference by fixing $\mathrm{CN}$ as the known crystallographic value. 
Table S4. ORR activity comparison of IrCo-N-C with the reported atomically dispersed M-N-C catalysts.

\begin{tabular}{cccc}
\hline Catalyst & Loading $\left(\mathrm{mg} \mathrm{cm}^{-2}\right)$ & $\mathrm{E}_{1 / 2}(\mathrm{~V})$ & Reference \\
\hline IrCo-N-C & 0.4 & 0.911 & This work \\
S,N-Fe/N/C-CNT & 0.6 & 0.85 & 9 \\
Fe/SNC & 0.5 & 0.86 & 10 \\
Co-ISAS/p-CN & - & 0.838 & 11 \\
Co-N/CNFs & 0.1 & 0.82 & 12 \\
Co SAs/N-C(900) & 0.408 & 0.881 & 13 \\
Co@MCM & 0.485 & 0.86 & 14 \\
Fe-N/C-800 & 0.079 & 0.81 & 15 \\
L-CCNTs-Co-800 & 0.198 & 0.84 & 16 \\
Pt 1 -N/BP & 0.39 & 0.87 & 17 \\
ZnNx/BP & 0.39 & 0.85 & 18 \\
Cu-N-C & 0.5 & 0.869 & 19 \\
\hline
\end{tabular}


Table S5. Zn-air battery performance comparison of IrCo-N-C with the reported atomically dispersed M-N-

C catalysts

\begin{tabular}{llll}
\hline Catalyst & Peak power density $\left(\mathrm{mW} \mathrm{cm}^{-2}\right)$ & $\begin{array}{l}\text { Voltage gap }(\mathrm{V} @ 5, \\
\left.\mathrm{mA} \mathrm{cm}{ }^{-2}\right)\end{array}$ & Reference \\
\hline IrCo-N-C & 167 & 0.701 \\
S,N-Fe/N/C-CNT & 102.7 & $\sim 0.75$ & 9 \\
CoNi-SAs/NC & 101.4 & 0.82 & 20 \\
Co-N,B-CSs & 100.4 & 1.35 & 21 \\
NGM-Co & 152 & 1.12 & 22 \\
sCu-ONPC & 88.5 & 0.83 & 23 \\
Co-POC & 78 & $1.04\left(@ 2 \mathrm{~mA} \mathrm{~cm}^{-2}\right)$ & 24 \\
Fe-Nx-C & 96.4 & 0.803 & 25 \\
CoSA/N,S-HCS & 173.1 & $0.82\left(@ 10 \mathrm{~mA} \mathrm{~cm}^{-2}\right)$ & 26 \\
Co-N $/ \mathrm{NC}$ & 101.62 & $1.16\left(@ 10 \mathrm{~mA} \mathrm{~cm}^{-2}\right)$ & 27 \\
\hline
\end{tabular}

Table S6 Calculated Ir-Co bond distance and the formation energies $\left(\mathrm{E}_{\mathrm{f}}\right)$ for the optimized configurations.

\begin{tabular}{lll}
\hline Structure & Metal distance $(\AA)$ & $\mathrm{E}_{\mathrm{f}}(\mathrm{eV})$ \\
\hline $\mathrm{IrCoN}_{6}-1$ & 2.305 & -7.67 \\
$\mathrm{IrCoN}_{6}-2$ & 2.295 & -5.38 \\
$\mathrm{IrCoN}_{5}$ & 2.343 & -6.59 \\
$\mathrm{IrN}_{4}$ & & -4.42 \\
$\mathrm{CoN}_{4}$ & & -3.92 \\
\hline
\end{tabular}


Table S7 Calculated Co-3d spin magnetic moment and Gibbs free adsorption energy of oxygen, $\Delta \mathrm{G}(\mathrm{O})$ on the as-built configurations.

\begin{tabular}{lll}
\hline Structure & Co-3d spin magnetic moment $\left(\mu_{\mathrm{B}}\right)$ & $\Delta \mathrm{G}(\mathrm{O})(\mathrm{eV})$ \\
\hline $\mathrm{IrCoN}_{6}-1$ & 0 & 3.35 \\
$\operatorname{IrCoN}_{6}-2$ & 2.12 & 0.41 \\
$\mathrm{IrCoN}_{5}$ & 1.80 & 1.95 \\
$\mathrm{CoN}_{4}$ & 0.9 & 3.01 \\
\hline
\end{tabular}

Table S8 The component of atomic orbital of Co and O in Co-O molecular orbital.

\begin{tabular}{ccc}
\hline & $\mathrm{CoN}_{4}$ & $\operatorname{IrCoN}_{5}$ \\
\hline $\mathrm{Co}(4 \mathrm{~s})-\mathrm{O}(2 \mathrm{~s})$ & $25.53 \%$ & $23.36 \%$ \\
$\mathrm{Co}(4 \mathrm{~s})-\mathrm{O}(2 \mathrm{p})$ & $3.38 \%$ & $3.41 \%$ \\
$\mathrm{Co}(3 \mathrm{~d})-\mathrm{O}(2 \mathrm{~s})$ & $3.92 \%$ & $4.90 \%$ \\
$\mathrm{Co}\left(3 \mathrm{~d}_{\mathrm{xy}}\right)-\mathrm{O}(2 \mathrm{p})$ & $0.01 \%$ & $1.83 \%$ \\
$\mathrm{Co}\left(3 \mathrm{~d}_{\mathrm{yz}}\right)-\mathrm{O}(2 \mathrm{p})$ & $14.79 \%$ & $18.43 \%$ \\
$\mathrm{Co}\left(3 \mathrm{~d}_{\mathrm{z} 2}\right)-\mathrm{O}(2 \mathrm{p})$ & $32.04 \%$ & $26.76 \%$ \\
$\mathrm{Co}\left(3 \mathrm{~d}_{\mathrm{xz}}\right)-\mathrm{O}(2 \mathrm{p})$ & $20.32 \%$ & $21.39 \%$ \\
$\mathrm{Co}\left(3 \mathrm{~d}_{(\mathrm{x} 2-\mathrm{y} 2}\right)-\mathrm{O}(2 \mathrm{p})$ & $0.00 \%$ & $-0.07 \%$ \\
\hline
\end{tabular}

Table S9 Integrated crystal orbital Hamilton populations (ICOHP) of the Co-O bond.

\begin{tabular}{lll}
\hline Structure & Spin state & ICOHP \\
\hline $\mathrm{CoN}_{4}$ & Spin up & -1.28 \\
\cline { 2 - 3 } & Spin down & -2.69 \\
\hline $\mathrm{IrCoN}_{5}$ & Spin up & -1.43 \\
& Spin down & -3.53 \\
\hline
\end{tabular}

Reference

1. Ravel, B.; Newville, M., ATHENA, ARTEMIS, HEPHAESTUS: data analysis for X-ray absorption spectroscopy using IFEFFIT. J Synchrotron Radiat 2005, 12, 537-541. 
2. Perdew, J. P. B., K.; Ernzerhof M, Generalized gradient approximation made simple Phys. Rev. Lett 1996, 77, 3865-3868.

3. Kresse, G.; Furthmuller, J., Efficiency of ab-initio total energy calculations for metals and semiconductors using a plane-wave basis set. Comp Mater Sci 1996, 6 (1), 15-50.

4. Kresse, G.; Hafner, J., Ab initio molecular dynamics for liquid metals. Phys Rev B 1993, 47 (1), 558561.

5. Kresse, G.; Hafner, J., Ab initio molecular-dynamics simulation of the liquid-metal--amorphoussemiconductor transition in germanium. Phys Rev B 1994, 49 (20), 14251-14269.

6. Kresse, G.; Furthmüller, J., Efficient iterative schemes for ab initio total-energy calculations using a plane-wave basis set. Phys Rev B 1996, 54 (16), 11169-11186.

7. Monkhorst, H. J.; Pack, J. D., Special points for Brillouin-zone integrations. Phys Rev B 1976, 13 (12), $5188-5192$.

8. Grimme, S.; Antony, J.; Ehrlich, S.; Krieg, H., A consistent and accurate ab initio parametrization of density functional dispersion correction (DFT-D) for the 94 elements H-Pu. The Journal of chemical physics 2010, $132(15), 154104$.

9. Chen, P.; Zhou, T.; Xing, L.; Xu, K.; Tong, Y.; Xie, H.; Zhang, L.; Yan, W.; Chu, W.; Wu, C.; Xie, Y., Atomically Dispersed Iron-Nitrogen Species as Electrocatalysts for Bifunctional Oxygen Evolution and Reduction Reactions. Angew. Chem. Int. Ed. 2017, 56 (2), 610-614.

10. Shen, H.; Gracia-Espino, E.; Ma, J.; Zang, K.; Luo, J.; Wang, L.; Gao, S.; Mamat, X.; Hu, G.; Wagberg, T.; Guo, S., Synergistic Effects between Atomically Dispersed $\mathrm{Fe}-\mathrm{N}-\mathrm{C}$ and $\mathrm{C}-\mathrm{S}-\mathrm{C}$ for the Oxygen Reduction Reaction in Acidic Media. Angew. Chem. Int. Ed. 2017, 56 (44), 13800-13804.

11. Han, A.; Chen, W.; Zhang, S.; Zhang, M.; Han, Y.; Zhang, J.; Ji, S.; Zheng, L.; Wang, Y.; Gu, L.; Chen, C.; Peng, Q.; Wang, D.; Li, Y., A Polymer Encapsulation Strategy to Synthesize Porous Nitrogen-Doped Carbon-Nanosphere-Supported Metal Isolated-Single-Atomic-Site Catalysts. Adv. Mater. 2018, 30 (15), 1706508

12. Cheng, Q.; Yang, L.; Zou, L.; Zou, Z.; Chen, C.; Hu, Z.; Yang, H., Single Cobalt Atom and N Codoped Carbon Nanofibers as Highly Durable Electrocatalyst for Oxygen Reduction Reaction. ACS Catal. 2017, 7 (10), 6864-6871.

13. Yin, P.; Yao, T.; Wu, Y.; Zheng, L.; Lin, Y.; Liu, W.; Ju, H.; Zhu, J.; Hong, X.; Deng, Z.; Zhou, G.; Wei, S.; Li, Y., Single Cobalt Atoms with Precise N-Coordination as Superior Oxygen Reduction Reaction 
Catalysts. Angew. Chem. Int. Ed. 2016, 55 (36), 10800-10805.

14. Zhang, H.; Zhou, W.; Chen, T.; Guan, B. Y.; Li, Z.; Lou, X. W., A modular strategy for decorating isolated cobalt atoms into multichannel carbon matrix for electrocatalytic oxygen reduction. Energy Environ. Sci. 2018, $11(8), 1980-1984$.

15. Niu, W.; Li, L.; Liu, X.; Wang, N.; Liu, J.; Zhou, W.; Tang, Z.; Chen, S., Mesoporous N-Doped Carbons Prepared with Thermally Removable Nanoparticle Templates: An Efficient Electrocatalyst for Oxygen Reduction Reaction. J. Am. Chem. Soc. 2015, 137 (16), 5555-5562.

16. Liang, Z.; Fan, X.; Lei, H.; Qi, J.; Li, Y.; Gao, J.; Huo, M.; Yuan, H.; Zhang, W.; Lin, H.; Zheng, H.; Cao, R., Cobalt-Nitrogen-Doped Helical Carbonaceous Nanotubes as a Class of Efficient Electrocatalysts for the Oxygen Reduction Reaction. Angew. Chem. 2018, 130 (40), 13371-13375.

17. Liu, J.; Jiao, M.; Lu, L.; Barkholtz, H. M.; Li, Y.; Wang, Y.; Jiang, L.; Wu, Z.; Liu, D.-j.; Zhuang, L.; Ma, C.; Zeng, J.; Zhang, B.; Su, D.; Song, P.; Xing, W.; Xu, W.; Wang, Y.; Jiang, Z.; Sun, G., High performance platinum single atom electrocatalyst for oxygen reduction reaction. Nat. Commun. 2017, 8, 15938.

18. Song, P.; Luo, M.; Liu, X.; Xing, W.; Xu, W.; Jiang, Z.; Gu, L., Zn Single Atom Catalyst for Highly Efficient Oxygen Reduction Reaction. Adv. Funct. Mater. 2017, 27 (28), 1700802.

19. Li, F.; Han, G.-F.; Noh, H.-J.; Kim, S.-J.; Lu, Y.; Jeong, H. Y.; Fu, Z.; Baek, J.-B., Boosting oxygen reduction catalysis with abundant copper single atom active sites. Energy Environ. Sci. 2018, 11 (8), 22632269.

20. Han, X.; Ling, X.; Yu, D.; Xie, D.; Li, L.; Peng, S.; Zhong, C.; Zhao, N.; Deng, Y.; Hu, W., Atomically Dispersed Binary Co-Ni Sites in Nitrogen-Doped Hollow Carbon Nanocubes for Reversible Oxygen Reduction and Evolution. Adv. Mater. 2019, 31 (49), 1905622.

21. Guo, Y.; Yuan, P.; Zhang, J.; Hu, Y.; Amiinu, I. S.; Wang, X.; Zhou, J.; Xia, H.; Song, Z.; Xu, Q.; Mu, S., Carbon Nanosheets Containing Discrete Co-Nx-By-C Active Sites for Efficient Oxygen Electrocatalysis and Rechargeable Zn-Air Batteries. Acs Nano 2018, 12 (2), 1894-1901.

22. Tang, C.; Wang, B.; Wang, H.-F.; Zhang, Q., Defect Engineering toward Atomic $\mathrm{Co}-\mathrm{Nx}-\mathrm{C}$ in Hierarchical Graphene for Rechargeable Flexible Solid Zn-Air Batteries. Adv. Mater. 2017, 29 (37), 1703185.

23. Wang, Y.; Jin, M.; Zhang, X.; Zhao, C.; Wang, H.; Li, S.; Liu, Z., Direct Conversion of Biomass into Compact Air Electrode with Atomically Dispersed Oxygen and Nitrogen Coordinated Copper Species for 
Flexible Zinc-Air Batteries. ACS Applied Energy Materials 2019, 2 (12), 8659-8666.

24. Li, B.-Q.; Zhao, C.-X.; Chen, S.; Liu, J.-N.; Chen, X.; Song, L.; Zhang, Q., Framework-PorphyrinDerived Single-Atom Bifunctional Oxygen Electrocatalysts and their Applications in Zn-Air Batteries. Adv. Mater. 2019, 31 (19), 1900592.

25. Han, J.; Meng, X.; Lu, L.; Bian, J.; Li, Z.; Sun, C., Single-Atom Fe-Nx-C as an Efficient Electrocatalyst for Zinc-Air Batteries. Adv. Funct. Mater. 2019, 29 (41), 1808872.

26. Zhang, Z.; Zhao, X.; Xi, S.; Zhang, L.; Chen, Z.; Zeng, Z.; Huang, M.; Yang, H.; Liu, B.; Pennycook, S. J., Atomically Dispersed Cobalt Trifunctional Electrocatalysts with Tailored Coordination Environment for Flexible Rechargeable Zn-Air Battery and Self-Driven Water Splitting. Adv. Energy Mater. 2020, 10 (48), 2002896.

27. Chen, K.; Kim, S.; Je, M.; Choi, H.; Shi, Z.; Vladimir, N.; Kim, K. H.; Li, O. L., Ultrasonic Plasma Engineering Toward Facile Synthesis of Single-Atom M-N4/N-Doped Carbon $(\mathrm{M}=\mathrm{Fe}, \mathrm{Co})$ as Superior Oxygen Electrocatalyst in Rechargeable Zinc-Air Batteries. Nano-Micro Letters 2021, 13 (1), 60. 